\title{
Educación para la paz como insumo para el posconflicto en Colombia
}

\section{Peace education as a resource for Colombia's post conflict}

\author{
Eyesid Álvarez Bahena*, Claudia Nayibe Sánchez
}

Moncayo $^{* *}$, Juan Diego Vélez Puerta ${ }^{* * *}$

Recibido: 25 de noviembre de 2020 / Aceptado: 8 de julio de 2021 / Publicado: 13 de diciembre de 2021

\section{Forma de citar este artículo en APA:}

Álvarez Bahena, E., Sánchez Moncayo, C. N., \& Vélez Puerta, J. (2021). Educación para la paz como insumo para el posconflicto en Colombia. Poiésis (41), 80-92. https://doi.org/10.21501/16920945.4176

\section{Resumen}

Este artículo resultado de investigación tiene como objetivo examinar las competencias ciudadanas que están desarrollando los estudiantes en su proceso formativo de la Universidad Católica Luis Amigó para una cultura de paz. La metodología empleada fue la investigación cualitativa con un enfoque interpretativo a través de un estudio de caso. De acuerdo con los resultados, se identificó que los estudiantes definen las competencias ciudadanas como la habilidad de saber habitar y ser parte de una sociedad, asociándose en efecto con el sentido de pertenencia a la misma y con que permite la interacción entre personas, ello desde una mirada holística de lo que está permitido o no en una sociedad. Finalmente, la formación en competencias ciudadanas y una educación para la paz son elementos determinantes para la construcción de una cultura de paz, en la medida que estas fortalecen las interacciones sociales en una sociedad fragmentada por las violencias durante décadas.

\section{Palabras clave:}

Ambiente de la clase; Cultura de paz; Educación ciudadana; Educación para la paz; Formación política. 


\section{Abstract}

This research result article aims to examine the civic competencies that students are developing in their formative process at Luis Amigo Catholic University for a culture of peace. The methodology used was qualitative research with an interpretive approach through a case study. According to the results, students define citizenship competencies as the ability to know how to live and be part of a society, associating in effect with the sense of belonging to it and allowing interaction between people, analyzing from a holistic perspective what is allowed or not in a society. Finally, training in citizenship skills and education for peace are determining elements for the construction of a culture of peace, insofar as they strengthen social interactions in a society fragmented by violence for decades.

\section{Keywords:}

Class environment; Peace culture; Civic education; Education for peace; Political formation. 


\section{Introducción}

Para el fin de la confrontación armada, el Estado y la sociedad civil en Colombia históricamente han generado iniciativas de paz; sin embargo, Chernick (1999) argumenta que estas se han desarrollado en múltiples violencias, inicialmente están los procesos de negociación en el gobierno de Belisario Betancur (1982-1986), luego Virgilio Barco (1986-1990), César Gaviria (1990-1994), Ernesto Samper Pizano (1994-1998), Andrés Pastrana (1998-2002), Álvaro Uribe Vélez (2002- 2010) y el gobierno de Juan Manuel Santos (2010-2018).

En una época de posconflicto, la implementación de una cultura de paz a través de la educación es fundamental para el proceso de reconstrucción de la sociedad, la conservación de la memoria y la creación de cultura e identidad; por lo cual, el desarrollo de competencias ciudadanas a través de esta es trascendental.

Como resultado se espera analizar las competencias ciudadanas que están desarrollando los estudiantes del último semestre de la Facultad de Educación de la Universidad Católica Luis Amigó para una cultura de paz, identificándolas y describiéndolas.

Dado que la intención de este artículo está dirigida hacia el entendimiento de la educación para la paz como insumo para la construcción de una cultura de paz en el contexto de postconflicto colombiano, es necesario plantear algunos parámetros que sirven de ejes conceptuales para la lectura interpretativa del texto. Para empezar, se entiende el concepto de paz no como la ausencia de guerra, sino desde las causas sociales, políticas y económicas que la generan (Villamil Cancino, 2013). En esta misma perspectiva, se denomina paz positiva a esta capacidad de actuar y mediar en la resolución pacífica de las diferencias, al igual que a la satisfacción de necesidades de seguridad, bienestar, libertad e identidad (López Becerra, 2011). Así pues, la comprensión de la paz ha de tenerse como sinónimo de justicia, equidad y tolerancia, para llegar de esta forma al inicio del cambio del sistema cultural y sistema educativo. En esta línea, el concepto de paz debe:

Propender por una perspectiva alternativa encaminada a la intervención eficaz basada en el aprendizaje y análisis de las situaciones conflictivas, orientada al descubrimiento de su complejidad y multidimensionalidad, cuyo objetivo será favorecer el encuentro de salidas no violentas y consolidar escenarios educativos de construcción de cultura de paz. (Areiza Madrid, 2014, p. 319)

Molano et al. (2008) precisan que la cultura de paz se fundamenta en actitudes, comportamientos y valores que estimulan el reconocimiento y aceptación de la diferencia, al igual que los derechos y libertades fundamentales de cada persona. En la cultura de paz es preciso señalar que se destacan como valores la igualdad, la libertad, el respeto, el amor y la paz. Por su parte, Sánchez Cardona (2012) entiende la cultura de paz como una práctica en pro de una sociedad con equidad, justicia, entre otras características; por tanto, si se educa a la ciudadanía desde una perspectiva 
de paz, es posible que esta pueda encontrar soluciones a los conflictos por vías no violentas. Además, desde Vila Merino y Martín Solbes (2011), se puede percibir que en la cultura de paz no se busca la dominación del otro, ya que esta se fundamenta en el respeto y reconocimiento de la diversidad, pues comprender la pluralidad humana es fundamental en el ejercicio mancomunado de la cultura de paz y la filosofía de la educación. En esta misma perspectiva, Cano y Molina Rueda (2015) expresan que la cultura de paz es la presencia de mecanismos 0 alternativas que fomentan el diálogo, que ayudan a establecer consensos y hallar valores en común.

Méndez Méndez y Casas Casas (2009) presentan las competencias ciudadanas como un mecanismo relevante en la construcción de cultura de paz, es decir que, para el desarrollo de conductas a favor de la resolución no violenta de los conflictos, es necesario trabajar en la comprensión de competencias sociales como el respeto y tolerancia a la diferencia, entre otros. Además, como la escuela reproduce las relaciones de poder y dominaciones dadas en las esferas sociales, por tanto, la escuela debe construirse como un espacio de desnaturalización de las injusticias (Botero et al., 2008). Asimismo, Hernández Arteaga et al. (2017) sustentan que:

La educación para una cultura de paz en la actualidad, es una educación para la vida, que implica ser, conocer, hacer y convivir mediante el respeto de los derechos humanos y el medio ambiente. Una educación que cultive en el ser humano los valores de justicia, equidad, solidaridad, libertad, autonomía, entre otros; pero a la vez, tenga la autoridad para criticar y cuestionar valores contrarios al desarrollo de una cultura de la paz, que requiere que todos los actores implicados desaten procesos de paz. (p. 168)

En esta misma dirección, es aceptable pensar que, si se educa a la ciudadanía desde una perspectiva de paz, es posible que esta pueda encontrar soluciones a los conflictos por vías no violentas.

Guajardo (2013) explica que usualmente se piensa que hay una diferencia entre educación para la paz y construcción de la paz, por ello es necesario aclarar que la educación para la paz intenta cambiar las conductas de la gente, mientras que la construcción de paz implica la incorporación de la justicia social y económica. Martínez Lirola (2015) anuncia que la educación para la paz ofrece tanto el favorecimiento de la enseñanza de valores en el proceso de enseñanza-aprendizaje, como propuestas para la prevención de conflictos o para su resolución de forma pacífica. Por su parte Hernández Arteaga et al. (2017) apuntan a que la educación para la paz debe ocuparse de las acciones a tomar respecto a la guerra y los conflictos, al igual que desarrollar competencias para actuar en pro de la paz; estas competencias son: mediar en la resolución de conflictos, conciliar las partes en desacuerdo, empatizar con las distintas partes, y construir ambientes de solidaridad.

De allí, entonces, Martínez Lirola (2016) propone que un objetivo esencial de la educación para la paz sea la intervención en la construcción de competencias ciudadanas en pro de la resolución pacífica de los conflictos y la sana convivencia. Argumento apoyado también por Cerdas-Agüero (2015), quien además piensa que la educación para la paz dota al individuo de estrategias por 
medio de las cuales han de promover y defender los derechos humanos, y principalmente, posibilitar la práctica constante de ellos. Además, esta debe de ir en pro de la construcción de un sujeto solidario y para la vida.

Hernández Arteaga et al. (2017) opinan que la educación para la paz es un proceso dirigido al cambio de personalidad constante, cuyo fin ha de ser la posibilidad de convivir con los demás en un entorno de solidaridad y justicia social.

\section{Metodología}

Este estudio ha sido realizado bajo la metodología de investigación cualitativa, la cual a través de Sandoval (1997) puede entenderse como un método cuyo interés apunta, especialmente, a comprender la realidad social como parte del resultado de un proceso histórico, analizando desde una perspectiva de la lógica o el sentido común y sobre todo, desde los actores.

En consecuencia de lo anterior, la selección del modelo de investigación cualitativo se hizo porque este se funda en una perspectiva interpretativa centrada en el entendimiento del significado de las acciones de los actores, es decir, porque busca interpretar lo que va captando de forma activa, y así poder comprender el conflicto como fenómeno y, a su vez, percibir posibles soluciones mediante la cultura de paz y la educación como mediadores en la resolución de conflictos.

Por la naturaleza del fenómeno estudiado, se eligió la estrategia de investigación de estudio de caso, en la cual, a partir de la observación detallada de una experiencia, se puede mostrar las características de actores, escenarios y contextos que pueden dar cuenta de una realidad particular (Hernández Sampieri \& Mendoza, 2008). Con base en lo dicho, este estudio de caso está dirigido a analizar las competencias ciudadanas que están desarrollando los estudiantes de la Universidad Católica Luis Amigó para una cultura de paz.

Se empleó el enfoque metodológico hermenéutico comprensivo, el cual, advierte Gadamer (1960/2001), posibilita la interpretación de la subjetividad del actor, dado a que el sujeto que se analiza es en sí mismo un lenguaje, el cual debe ser entendido e interpretado con el contexto y las categorías de apoyo que el lenguaje proporciona.

Como instrumento para la recolección de la información se utilizó la entrevista estructurada realizada a ocho estudiantes de la facultad de educación de la Universidad Católica Luis Amigó sede Medellín, que cursan el último semestre de su carrera de pregrado, con edades comprendidas entre los 20 y 36 años. En estos diálogos se les preguntó sobre su concepción y conocimiento acerca de las competencias ciudadanas, cultura de paz, estrategias empleadas para la resolución de conflictos, prácticas de paz; ello con el fin de aprehender el conocimiento de mecanismos y capacidad de imaginar distintas formas de resolución de conflictos, al igual que el conocimiento y manejo de las emociones de los estudiantes. 
Para el análisis de calidad de las entrevistas fue creada una matriz en el software Microsoft Word 2007, cuya estructura estuvo determinada por: declaración de la persona entrevistada, categorías de análisis (a saber: 1. Concepción de competencias ciudadanas, 2. Tipos de competencias ciudadanas, 3. Cultura de paz), subcategorías y comentarios.

La elaboración de este artículo se realizó en varios momentos; el primero fue el de exploración, en el cual se realizó el acercamiento a nociones y fuentes informativas con base en técnicas e instrumentos que permitan poder conocer la noción del fenómeno de la cultura de paz y competencias ciudadanas, también se efectuó el anteproyecto de investigación y la elaboración de las fichas de contenido. El segundo momento fue el de focalización, en el cual se realizó el diseño metodológico, además del diseño de preguntas y la realización de entrevistas estructuradas como instrumentos de generación de la información. El tercer momento fue el proceso de profundización y análisis de la información; la técnica de registro fue la transcripción de las entrevistas realizadas a los estudiantes de último semestre de la facultad de educación, identificación de categorías de análisis, codificación y el posterior análisis a través de la interpretación de matrices.

\section{Resultados}

\section{Concepción de los estudiantes sobre las competencias ciudadanas}

Para efectos de este trabajo, desde el Ministerio de Educación Nacional (MEN, 2015), una competencia ciudadana es entendida como el conjunto de conocimientos, habilidades y actitudes que desarrollan las personas, que les permiten comprender, interactuar y transformar el mundo en el que viven. En este orden de ideas, se identificó en los estudiantes sujetos de estudio que las definen como la destreza de saber habitar y ser parte de una sociedad, asociándolas en efecto con el sentido de pertenencia a la misma, así: "una competencia ciudadana es la capacidad de vivir adecuadamente en una ciudad, sintiéndome ciudadano, hay un sentido de pertenencia" (Entrevistado 1).

Admiten además la interacción entre personas, pudiendo permitiendo tener una mirada holística de lo que se está autorizando o no en una sociedad. Así lo expresan los estudiantes en sus declaraciones: "Como una habilidad que nos permite interactuar con el otro" (Entrevistado 5). "Me voy también por el ámbito de la habilidad, que nos ayuda a ser capaces de entender dentro de las comunidades, de la sociedad, lo que hay y lo que se puede hacer dentro de ella" (Entrevistado 6). "Yo lo veo más como las pautas que permiten al ciudadano socializar con el resto de su comunidad y como poderse desenvolver en dicho contexto" (Entrevistado 7).

Igualmente son relacionadas con los mecanismos de participación ciudadana como el voto, con el respeto de las normas de tránsito y se resalta que por medio de estas se puede mantener el orden social, estimular la buena convivencia y las relaciones interpersonales para el bienestar de los habitantes de una comunidad. 
A partir de allí, los estudiantes resaltan normas y valores de urbanidad, asociándolos a tipos de competencias ciudadanas, tales como el respeto a las normas de tránsito, la cultura metro y la señalización de depósitos de basura, entre otros. Siendo además la escucha, el respeto y pedir la palabra, las competencias más empleadas en espacios de discusión; como también la comunicación asertiva, la solidaridad, el autocuidado y el cuidado del medio ambiente: "si he de participar en algo que yo tengo un punto de vista diferente, primero es como pedir la palabra y sin ánimos pues como de pelear, siempre respetando la opinión" (Entrevistado 3). "El diálogo, la comunicación asertiva, las señales que hay dentro de los espacios, digamos en la universidad las señalizaciones, las personas que nos pueden colaborar" (Entrevistado 6). "Practico por ejemplo las normas de tránsito, como el respeto a los semáforos, también creo que otra de las competencias es el cuidado del medio ambiente, podría ser, entonces trato de ser cuidadosa con el medio ambiente, no tirar las basuras al piso" (Entrevistado 1). "No sé si de pronto aplique las normas que uno debe tener en el 'Metro', 'cultura metro', o de pronto también las señales de basura, pero sí, todo eso lleva más como al respeto" (Entrevistado 3).

\section{Competencias ciudadanas de los estudiantes y cultura de paz}

Entre las competencias ciudadanas que la Universidad está formando en el estudiante se hallaron la autonomía, la formación e innovación en el saber pedagógico, estimulando a través de los docentes el desarrollo principalmente de profesionales éticos y de competencias como el respeto hacia los demás y a la norma.

Desde la Universidad creo que fomentando y creando estos espacios para las competencias ciudadanas, por ejemplo, los espacios también desde los semilleros, uno puede plantear también muchas iniciativas que ayudan para el bien común, creo que también a nivel académico, la ética, la humanidad de los profesores, de los alumnos, todo esto genera un ambiente de paz, desde lo pequeño desde este pequeño mundo yo puedo crear un ambiente de paz. Me parece una Universidad muy humana. (Entrevistado 1)

Desarrollar de pronto esa autonomía en el estudiante, de pronto aquí en la cátedra que nos han dado y más que todo en la licenciatura, es más que todo cambiar ese paradigma de ser esa persona 0 esos profesores tradicionales, entonces como ya ser un poco más innovador. (Entrevistado 4)

Entre las competencias ciudadanas que practican los estudiantes para la resolución de conflictos, se identificaron el diálogo, la comunicación asertiva y la imparcialidad: "pienso en ser equitativo a la hora de resolver ciertos conflictos" (Entrevistado 2). "Uno al irrespeto debe responder con respeto, yo me considero muy calmado (...) de pronto en ese momento no tendría ninguna reacción agresiva o negativa, pues también puedo salir yo perjudicado" (Entrevistado 4).

No obstante, la práctica de estas competencias también está mediada por la situación que se enfrente, pues dependiendo del contexto puede variar el grado de comunicación asertiva en la manifestación de su inconformidad, pero haciendo la salvedad de que no se llega a hechos de agresión o violencia: 
Si es en un momento de alteración, vas a manifestarlo de una manera dominante, pero si uno hace primero una reflexión, piensa bien las cosas, yo creo que ya se puede dar una mediación, un diálogo como mucho más acorde; yo creo que depende mucho de la situación. (Entrevistado 6)

"En muchas ocasiones no se da el diálogo, sino que muchas veces se busca como estar tranquilas o mejorar lo que le disgusto para más adelante poderlo hablar" (Entrevistado 5).

En esta misma perspectiva, para la resolución de un conflicto, la reflexividad se manifiesta como una alternativa previa o como mecanismo que le sirve al estudiante para examinar bien la situación, aceptar su error y para proceder con la conciliación con la otra parte ofreciendo disculpas. No obstante, la "reparación" o acciones en beneficio del otro son otras alternativas para ofrecer disculpas percibidas en los estudiantes.

Reconocer un error siempre va a ser muy difícil, pero igual, en el momento en que uno lo hace también está como la madurez y el criterio de asumirlo y pedir disculpas, o si no se da la oportunidad de pedir disculpas, al menos tener acciones que demuestren un cambio a ese comportamiento erróneo. (Entrevistado 8)

De igual forma se inclinan a tener acciones evasivas como herramienta para la resolución de un conflicto, lo que permite fortalecer la competencia comunicativa, pues se puede posponer la discusión para un momento de calma en el que se pueda conversar con más asertividad.

Hablando con esa persona, o si otro me lo hace ver, agradezco; si otra persona me dice mira te equivocaste en esto puede que yo en un primer momento reflexiones bien y diga bueno me equivoqué. Trato de mejorar en lo que la persona me dijo que estaba mal, me esfuerzo por ser mejor. (Entrevistado 1)

La práctica de las competencias ciudadanas estimula la creación de una cultura de paz, advirtiendo que para ello es tanto necesario ir al encuentro del otro ante una dificultad, como la escucha, la comunicación asertiva, el diálogo, la tolerancia, el respeto y la reflexividad; son competencias ciudadanas que el estudiante también asocia a prácticas de paz y a las que suman el tener una buena actitud, la cortesía y el buen trato a los demás: "una práctica de paz es ir al encuentro del otro cuando hay una dificultad, escuchar, plantear mis opiniones, mis maneras de ver las cosas... ser delicado con el otro, buscar el diálogo" (Entrevistado 1).

Yo pienso que uno siempre debe tener la buena actitud, tratar de uno llevar... si uno tiene un día malo tratar de ser muy neutro, tener casi siempre un buen ambiente, para que en realidad se pueda aplicar una cultura de paz. Yo lo veo desde mi personalidad, no soy muy sociable, pero sí soy muy buen compañero, te saludo a ti y a cualquiera. (Entrevistado 4)

Yo me seguiría enfocando en el respeto, puede ser, respecto a ser puntuales, responsables, respetar al otro, respetar el turno, la palabra, escuchar. (...) eso es algo con lo que uno viene también, pero también hay ciertas clases y profesores que promueven eso. (Entrevistado 3) 
La cultura de paz se funda en actitudes, comportamientos y valores que estimulan el reconocimiento y aceptación de la diferencia, al igual que los derechos y libertades fundamentales de cada persona, además de valores como la igualdad, la libertad, el respeto, el amor y la paz. Prácticas que van dirigidas al fortalecimiento de una sociedad con equidad y justicia, por lo cual cada ciudadano tiene la gran responsabilidad de hallar soluciones a los conflictos por vías no violentas: "de pronto como tener esa capacidad de que haya un ambiente sano, de equidad" (Entrevistado 3). "Esa cultura de la paz uno la concibe como la tranquilidad, de que no esté tanto en conflicto, que se cree un buen ambiente" (Entrevistado 4). "Sería esa cultura de saber vivir en comunidad y de estar también en paz con uno mismo y de llevar las cosas bien" (Entrevistado 2).

En esta misma perspectiva, los estudiantes de la Universidad Católica Luis Amigó asocian la cultura de paz con estados de tranquilidad, equidad, buena convivencia y ambientes sanos, que generan confort con sí mismo y los demás; hábitos que ayudan a la buena convivencia y generación de ambientes sanos y propicios para un bien común. Por ende, la construcción de una cultura de paz no es solo responsabilidad de la academia y del estado, sino también un compromiso de todos los ciudadanos.

La Universidad aporta a la formación de una cultura de paz, prácticas inclusivas y de solidaridad para las personas con movilidad reducida y discapacidad visual, realizando los ajustes razonables en el entorno y ayudas técnicas conforme a las necesidades de la población específica:

El hecho de tener personas con otras necesidades a las que se ven comúnmente en la sociedad, que necesitan el apoyo y la ayuda del otro ser, que se da una inclusión dentro de la Universidad y muchas veces vemos en los pasillos personas ciegas y muchas personas van y les ayudan, se tiene la disponibilidad de los salones para discapacitados. (Entrevistado 6)

La Universidad cuenta con cátedra sobre la paz y docentes que inspiran en el estudiante tranquilidad y el gusto por aprender, lo cual es un buen estímulo para la creación de una cultura de paz. Cabe anotar que desde su práctica universitaria, el estudiante aporta a la creación de una cultura de paz no creando divisiones, practicando la tolerancia a la diferencia, la honestidad acompañada de la comunicación asertiva directamente con la persona que se tiene la inconformidad, ello con el fin de evitar malas interpretaciones por terceros.

Aceptando al otro como es, también no creando división, porque en esos espacios hay el espacio de estudiar, pero también uno se encuentra en los corredores, en las clases, a tomar un café con el otro, entonces puede salir la crítica hacia el otro, hacia los mismos profesores... si a uno no le gusta algo de un profesor, sentarse uno mismo y decirle "esto está mal profe, yo creo o considero que le faltó esto", en vez de uno estar por fuera hablando del otro, haciendo juicio. (Entrevistado 1) 
Con respecto a la práctica pedagógica, los estudiantes dan ejemplo a los niños, enseñándoles conductas de paz asociadas a las normas cívicas como recoger y depositar las basuras en su lugar; resaltando también la importancia de que estas normas básicas deben ser implementadas desde la niñez, pues si se enseña a los niños buenos hábitos de convivencia, ello se replicará en las personas que les rodean y en futuras generaciones.

Primordialmente, nosotras como docentes tenemos como ese reto, la obligación de dar un ejemplo propicio a los niños, o sea, no darles una clase de simplemente ¿qué es la paz? ¿Qué es lo que se debe hacer o qué dice la norma?, sino que desde nosotras mismas qué le estamos impartiendo desde nuestro ser. (Entrevistado 7)

De allí, entonces, que un objetivo esencial de la educación para la paz sea la intervención en la construcción de competencias ciudadanas en pro de la resolución pacífica de los conflictos y la sana convivencia, orientada a la posibilidad de convivir con los demás en un entorno de solidaridad y justicia, ayudando a la enseñanza de valores en el proceso de enseñanza-aprendizaje.

Son como esas pautas que llegan como a un acuerdo con todos los ciudadanos para buscar ese objetivo que es la paz. Es la ayuda, la herramienta que se tiene para que se pueda vivir en una comunidad tranquila (...) en el ámbito educativo el bullying, es como buscar esos ambientes sanos y propicios para un bien común, y son como las acciones que todos llevamos a cabo para tener una sana convivencia. (Entrevistado 5)

\section{Discusión}

Los resultados de este estudio indican que el desarrollo de competencias ciudadanas mediante la educación es un buen estímulo para la creación de una cultura de paz, puesto que permite inculcar en el individuo capacidades no solo cognitivas, que develan un buen nivel de análisis crítico de las situaciones, si no además comunicativas como el diálogo, la escucha y la comunicación asertiva, que son herramientas mediadoras en la resolución pacífica de las diferencias. Esto es posible contrastarlo con investigaciones como la de Guajardo (2013), en la que es posible advertir que para una educación integral es necesario tener en cuenta los pilares de aprender a ser y aprender a convivir, lo cual nos conduce al reconocimiento de los derechos individuales y sociales al igual que a la formación en y para la convivencia armónica, solidaria y tolerante.

En esta misma perspectiva, la educación para la paz obtiene un reto concreto en el marco del posconflicto colombiano, expresado en que lo que se enseña y aprende en las universidades debe tener relación directa con lo que a bien demande la sociedad, dotando al alumno de una formación integral en pro de una configuración de ciudadanía activa (Martínez Lirola, 2015).

Resulta oportuno advertir que el cuidado del medio ambiente es una competencia ciudadana que estimula la creación de una cultura de paz, por lo cual es necesaria la reflexión en torno a esta perspectiva. Por tanto, las instituciones educativas colombianas deben fomentar la cultura de paz y nuevas acepciones de la paz misma, es decir, dotar al alumnado de herramientas que permitan 
la solución no violenta de los conflictos (Areiza Madrid, 2014). De allí, entonces, que un objetivo esencial de la educación para la paz sea la intervención en la construcción de competencias ciudadanas en pro de la resolución pacífica de los conflictos y la sana convivencia (Martínez Lirola, 2016).

\section{Conclusiones}

En la formación de los estudiantes de la Universidad Católica Luis Amigó como agentes constructores de una cultura de paz, es posible identificar que ellos se destacan por el desarrollo de competencias ciudadanas cognitivas que muestran buenos niveles de análisis crítico y reflexivo; $y$ por competencias emocionales que propenden por la empatía con las demás personas, el respeto, la tolerancia a la diferencia y competencias comunicativas, pues cuentan con gran capacidad de escucha, comunicación asertiva y suelen acudir a acciones evasivas, asistir al encuentro del otro y al diálogo como herramientas para la resolución pacífica de conflictos.

Educar para la paz no es una responsabilidad exclusivamente del Estado o el sector educativo; es también una cuestión de responsabilidad social y compromiso individual de generar ambientes de sana convivencia; es decir que es necesario un cambio de la educación tradicional, hacia una educación dirigida a desarrollar competencias sociales para la vida, que posibiliten resolver los conflictos de una manera no violenta. Para ello, el docente ha de centrar su desempeño no solo en su saber fundante, sino también en una educación con miras al respeto, la tolerancia y la resolución no violenta de los conflictos.

La educación para la paz no debe quedarse solo en una actitud de obrar bien o de hacer cosas buenas, ya que a través de la enseñanza de competencias ciudadanas se puede también estimular un compromiso político con el otro y la capacidad de expresión 0 argumentación de las razones para el bien común y, de esta forma, provocar espacios de cultura de paz.

\section{Conflicto de intereses}

Los autores declaran la inexistencia de conflicto de interés con institución o asociación comercial de cualquier índole. 


\section{Referencias}

Areiza Madrid, G. (2014). La educación para la paz en Colombia: estrategias de transformación y resolución negociada de los conflictos. Ra Ximhai, 10(4), 313-336. http://www.revistas.unam. $\mathrm{mx} /$ index.php/rxm/article/view/71205/62903

Botero, P., Ospina, H. F., Arcesio Gómez, E., \& Gutiérrez Ospina, M. (2008). Condiciones de participación y formación política de jóvenes colombianos constructores de paz. Revista Internacional de Investigación en Educación, 1(1), 81-93. https://revistas.javeriana.edu.co/index.php/ MAGIS/article/view/3357

Cano, M-J., \& Molina Rueda, B. (2015). Culturas, mundos globales y paz. Revista de Paz y Conflictos, 8(2), 9-27. https://revistaseug.ugr.es/index.php/revpaz/article/view/3178/3888

Cerdas-Agüero, E. (2015). Desafíos de la educación para la paz hacia la construcción de una cultura de paz. Revista Electrónica Educare, 19(2), 135-154. https://doi.org/10.15359/ree.19-2.9

Chernick, M. (1999). Negotiating peace amid multiple forms of violence: the protracted. In C. Arnson (Ed.), Search for a settlement to the armed conflicts in Colombia. Comparative peace processes in Latin America (pp. 93-100). Woodrow Wilson Center Press and Stanford University Press.

Gadamer, H-G. (2001). Verdad y método. Ediciones Sígueme. (Obra original publicado en 1960).

Guajardo, N. (2013). La educación para la paz y la mediación como herramientas en resolución de conflictos escolares. Espiral, Revista de Docencia e Investigación, 3(1), 45-56. https://doi. org/10.15332/erdi.v3i1.430

Hernández Arteaga, I., Luna Hernández, J., \& Cadena Chala, M. (2017). Cultura de paz: una construcción educativa aporte teórico. Revista Historia de la Educación Latinoamericana, 19(28), 149-172. https://doi.org/10.19053/01227238.5596

Hernández Sampieri, R., \& Mendoza, C. P. (2008). El matrimonio cuantitativo cualitativo: el paradigma mixto [Conferencia $6^{\circ}$ Congreso de Investigación en Sexología]. Instituto Mexicano de Sexología, A. C. \& Universidad Juárez Autónoma de Tabasco, Villahermosa, Tabasco, México.

López Becerra, M. (2011). Teorías para la paz y perspectivas ambientales del desarrollo como diálogos de imperfectos. Luna Azul, (33), 85-96.

Martínez Lirola, M. (2015). Aproximación a la educación para la paz en la enseñanza universitaria: Algunas actividades prácticas. Revista Científica Guillermo de Ockham, 13(2), 35-43. https:// revistas.usb.edu.co/index.php/GuillermoOckham/article/view/2062/1798 
Martínez Lirola, M. (2016). Potenciando el enfoque de la educación para la paz en la enseñanza universitaria española: una propuesta de actividades en asignaturas de lengua inglesa. Íkala, Revista de Lenguaje y Cultura, 22, 87-100. https://10.17533/udea.ikala.v22n01a06

Méndez Méndez, N., \& Casas Casas, A. (2009). Educación para la paz, cultura política y cambio social: un análisis empírico del programa Aulas en Paz desde el institucionalismo cognitivo. Desafíos, (21), 97-134. https://revistas.urosario.edu.co/index.php/desafios/article/view/448/390

Molano, 0., Campos, M., \& Quintero, M. (2008). Educación ciudadana universitaria para la cultura de paz. Multiciencias, 8(2), 163-171. https://produccioncientificaluz.org/index.php/multiciencias/ article/view/16696

Sánchez Cardona, M. (2012). Empoderamiento y responsabilidad de la cultura para la paz a través de la educación. Ra Ximhai, 8(2), 127-158.

Sandoval, C. (1997). Investigación Cualitativa. Módulo 4. Programa de Especialización en Teoría, Métodos y Técnicas de Investigación Social. ASCUN.

Vila Merino, E., \& Martín Solbes, V. (2011). Filosofía de la educación y cultura de paz en el discurso pedagógico. Innovación Educativa, 11(55), 6-13.

Villamil Cancino, M. (2013). Educación para la paz en Colombia: una búsqueda más allá del discurso. Espiral, Revista de Docencia e Investigación, 3(2), 25-40. https://doi.org/10.15332/ erdi.v3i2.460 